\title{
New congruences for sums involving Apéry numbers or central Delannoy numbers
}

\author{
Victor J. W. Guo and Jiang Zeng ${ }^{2}$ \\ ${ }^{1}$ Department of Mathematics, East China Normal University, \\ Shanghai 200062, People's Republic of China \\ jwguo@math.ecnu.edu.cn, http://math.ecnu.edu.cn/ ${ }^{j}$ wguo \\ ${ }^{2}$ Université de Lyon; Université Lyon 1; Institut Camille Jordan, UMR 5208 du CNRS; \\ 43, boulevard du 11 novembre 1918, F-69622 Villeurbanne Cedex, France \\ zeng@math.univ-lyon1.fr, http://math.univ-lyon1.fr/ zeng
}

\begin{abstract}
The Apéry numbers $A_{n}$ and central Delannoy numbers $D_{n}$ are defined by

$$
A_{n}=\sum_{k=0}^{n}\left(\begin{array}{c}
n+k \\
2 k
\end{array}\right)^{2}\left(\begin{array}{c}
2 k \\
k
\end{array}\right)^{2}, \quad D_{n}=\sum_{k=0}^{n}\left(\begin{array}{c}
n+k \\
2 k
\end{array}\right)\left(\begin{array}{c}
2 k \\
k
\end{array}\right) .
$$
\end{abstract}

Motivated by some recent work of Z.-W. Sun, we prove the following congruences:

$$
\sum_{k=0}^{n-1}(2 k+1)^{2 r+1} A_{k} \equiv \sum_{k=0}^{n-1} \varepsilon^{k}(2 k+1)^{2 r+1} D_{k} \equiv 0 \quad(\bmod n),
$$

where $n \geqslant 1, r \geqslant 0$, and $\varepsilon= \pm 1$. For $r=1$, we further show that

$$
\begin{aligned}
& \sum_{k=0}^{n-1}(2 k+1)^{3} A_{k} \equiv 0 \quad\left(\bmod n^{3}\right), \\
& \sum_{k=0}^{p-1}(2 k+1)^{3} A_{k} \equiv p^{3} \quad\left(\bmod 2 p^{6}\right),
\end{aligned}
$$

where $p>3$ is a prime. The following congruence

$$
\sum_{k=0}^{n-1}\left(\begin{array}{c}
n+k \\
k
\end{array}\right)^{2}\left(\begin{array}{c}
n-1 \\
k
\end{array}\right)^{2} \equiv 0 \quad(\bmod n)
$$

plays an important role in our proof.

Keywords: Apéry numbers, central Delannoy numbers, $q$-binomial coefficients, $q$-ChuVandermonde formula, $q$-Lucas theorem, Wolstenholme's theorem.

MR Subject Classifications: 11A07, 11B65, 05A10 


\section{Introduction}

The Apéry numbers [2] are given by

$$
A_{n}=\sum_{k=0}^{n}\left(\begin{array}{c}
n+k \\
2 k
\end{array}\right)^{2}\left(\begin{array}{c}
2 k \\
k
\end{array}\right)^{2} .
$$

Congruences for Apéry numbers were studied by many people, see Chowla et al. [7], Gessel [9], and Beukers [3], for example. The central Delannoy numbers (see [5, 13, 14]) are defined by

$$
D_{n}=\sum_{k=0}^{n}\left(\begin{array}{c}
n+k \\
2 k
\end{array}\right)\left(\begin{array}{c}
2 k \\
k
\end{array}\right) .
$$

Recently, Sun [14] has proved several remarkable congruences involving Apéry numbers or central Delannoy numbers. In this paper we will prove some similar congruences related to Apéry numbers and central Delannoy numbers, and establish some new congruences on sums of binomial coefficients and $q$-binomial coefficients. Let $\mathbb{N}$ denote the set of nonnegative integers and $\mathbb{Z}^{+}$the set of positive integers. Our main results may be stated as follows.

Theorem 1.1 Let $r \in \mathbb{N}$ and $n \in \mathbb{Z}^{+}$. Then

$$
\begin{aligned}
\sum_{k=0}^{n-1}(2 k+1) k^{r}(k+1)^{r} A_{k} & \equiv 0 \quad(\bmod n), \\
\sum_{k=0}^{n-1}(2 k+1)^{2 r+1} A_{k} & \equiv 0 \quad(\bmod n) .
\end{aligned}
$$

Theorem 1.2 Let $r \in \mathbb{N}$ and $n \in \mathbb{Z}^{+}$. Then

$$
\begin{array}{r}
\sum_{k=0}^{n-1} \varepsilon^{k}(2 k+1) k^{r}(k+1)^{r} D_{k} \equiv 0 \quad(\bmod n), \\
\sum_{k=0}^{n-1} \varepsilon^{k}(2 k+1)^{2 r+1} D_{k} \equiv 0 \quad(\bmod n),
\end{array}
$$

where $\varepsilon= \pm 1$.

When $r=0$ the above theorems were obtained by Sun [14]. For $r=1$, we have the following stronger result.

Theorem 1.3 Let $n \in \mathbb{Z}^{+}$, and let $p>3$ be a prime. Then

$$
\begin{aligned}
& \sum_{k=0}^{n-1}(2 k+1)^{3} A_{k} \equiv 0 \quad\left(\bmod n^{3}\right), \\
& \sum_{k=0}^{p-1}(2 k+1)^{3} A_{k} \equiv p^{3} \quad\left(\bmod 2 p^{6}\right) .
\end{aligned}
$$


In order to prove Theorem 1.3 we need to establish the congruence

$$
\sum_{k=0}^{n-1}\left(\begin{array}{c}
n+k \\
k
\end{array}\right)^{2}\left(\begin{array}{c}
n-1 \\
k
\end{array}\right)^{2} \equiv 0 \quad(\bmod n)
$$

In fact, we have the following more general result.

Theorem 1.4 Let $a_{1}, \ldots, a_{m}, b_{1}, \ldots, b_{m} \in \mathbb{N}$ and $n \in \mathbb{Z}^{+}$. Then

$$
\sum_{k=0}^{n-1}\left(\begin{array}{c}
n-1 \\
k
\end{array}\right)^{2} \prod_{i=1}^{m}\left(\begin{array}{c}
a_{i}+k \\
b_{i}+k
\end{array}\right) \equiv 0 \quad\left(\bmod \operatorname{gcd}\left(a_{1}, \ldots, a_{m}, b_{1}, \ldots, b_{m}, n\right)\right)
$$

We shall prove Theorems 1.1-1.4 in the next four sections, respectively, along with some conjectures for further studies.

\section{Proof of Theorem 1.1}

Let $(x)_{0}=1$ and $(x)_{n}=x(x+1) \cdots(x+n-1)$ for all $n \in \mathbb{Z}^{+}$. We first establish three lemmas.

Lemma 2.1 For all $k, m, r \in \mathbb{N}$, there exist $a_{0}(k, r), a_{1}(k, r), \ldots, a_{r}(k, r) \in \mathbb{Z}$ such that

$$
m^{r}(m+1)^{r}\left(\begin{array}{c}
m+k \\
2 k
\end{array}\right)=\sum_{j=0}^{r} a_{j}(k, r)\left(\begin{array}{c}
m+k+j \\
2 k+2 j
\end{array}\right)(2 k+1)_{2 j} .
$$

Proof. Given $k, r \in \mathbb{N}$, it is easy to see that there exist integers $a_{0}(k, r), a_{1}(k, r), \ldots, a_{r}(k, r)$, independent of $x$, such that

$$
x^{r}=\sum_{j=0}^{r} a_{j}(k, r) \prod_{i=1}^{j}(x-(k+i-1)(k+i)) .
$$

Substituting $x=m(m+1)$ in $(2.2)$, we get

$$
m^{r}(m+1)^{r}=\sum_{j=0}^{r} a_{j}(k, r)(m+k+1)_{j}(m-k-j+1)_{j}
$$

Multiplying both sides by $\left(\begin{array}{c}m+k \\ 2 k\end{array}\right)$ and noticing that

$$
\left(\begin{array}{c}
m+k \\
2 k
\end{array}\right)(m+k+1)_{j}(m-k-j+1)_{j}=\left(\begin{array}{c}
m+k+j \\
2 k+2 j
\end{array}\right)(2 k+1)_{2 j},
$$

we obtain (2.1). 
Lemma 2.2 For all $a, k, n \in \mathbb{N}$, there holds

$$
\sum_{m=k}^{n-1}(2 m+1)\left(\begin{array}{c}
m+k \\
2 k
\end{array}\right)\left(\begin{array}{c}
m+k+a \\
2 k+2 a
\end{array}\right)=\frac{(n-k)(n-k-a)}{2 k+a+1}\left(\begin{array}{c}
n+k \\
2 k
\end{array}\right)\left(\begin{array}{c}
n+k+a \\
2 k+2 a
\end{array}\right) .
$$

Lemma 2.2 can be proved easily by induction on $n$ (see Sun [14, Lemma 2.1] for the a=0 case). We can also evaluate the left-hand side of (2.3) automatically by Maple.

Lemma 2.3 For all $a, k \in \mathbb{N}$ and $n \in \mathbb{Z}^{+}$, there holds

$$
\frac{(n-k)(n-k-a)}{n(2 k+a+1)}\left(\begin{array}{c}
n+k \\
2 k
\end{array}\right)\left(\begin{array}{c}
n+k+a \\
2 k+2 a
\end{array}\right)\left(\begin{array}{c}
2 k \\
k
\end{array}\right)(2 k+1)_{2 a} \in \mathbb{N} .
$$

Proof. For $a=0$, we have

$$
\frac{(n-k)^{2}}{n(2 k+1)}\left(\begin{array}{c}
n+k \\
2 k
\end{array}\right)^{2}\left(\begin{array}{c}
2 k \\
k
\end{array}\right)=\left(\begin{array}{c}
n-1 \\
k
\end{array}\right)\left(\begin{array}{c}
n+k \\
k
\end{array}\right)\left(\begin{array}{c}
n+k \\
2 k+1
\end{array}\right) \in \mathbb{N} .
$$

For $a \geqslant 1$, we have

$$
\begin{aligned}
& \frac{(n-k)(n-k-a)}{n(2 k+a+1)}\left(\begin{array}{c}
n+k \\
2 k
\end{array}\right)\left(\begin{array}{c}
2 k \\
k
\end{array}\right)(2 k+1)_{2 a} \\
& =(n-k-a)\left(\begin{array}{c}
n+k \\
k
\end{array}\right)\left(\begin{array}{c}
n-1 \\
k
\end{array}\right)(2 k+1)_{a}(2 k+a+2)_{a-1} \in \mathbb{N} .
\end{aligned}
$$

This completes the proof.

Proof of Theorem 1.1. Substituting (1.1) for $A_{m}$ and applying Lemmas 2.1 and 2.2, we obtain

$$
\begin{aligned}
& \sum_{m=0}^{n-1}(2 m+1) m^{r}(m+1)^{r} A_{m} \\
& \quad=\sum_{j=0}^{r} \sum_{k=0}^{n-1} a_{j}(k, r)\left(\begin{array}{c}
2 k \\
k
\end{array}\right)^{2}(2 k+1)_{2 j} \sum_{m=k}^{n-1}(2 m+1)\left(\begin{array}{c}
m+k \\
2 k
\end{array}\right)\left(\begin{array}{c}
m+k+j \\
2 k+2 j
\end{array}\right) \\
& \quad=\sum_{j=0}^{r} \sum_{k=0}^{n-1} a_{j}(k, r)\left(\begin{array}{c}
2 k \\
k
\end{array}\right) C_{j}(k, n),
\end{aligned}
$$

where $a_{0}(k, r), a_{1}(k, r), \ldots, a_{r}(k, r)$ are integers determined by $(2.1)$ and

$$
C_{j}(k, n)=\left(\begin{array}{c}
2 k \\
k
\end{array}\right)(2 k+1)_{2 j} \frac{(n-k)(n-k-j)}{2 k+j+1}\left(\begin{array}{c}
n+k \\
2 k
\end{array}\right)\left(\begin{array}{c}
n+k+j \\
2 k+2 j
\end{array}\right) .
$$

By Lemma 2.3, we have $C_{j}(k, n) \equiv 0(\bmod n)$ and hence $(1.3)$ holds. Since

$$
(2 k+1)^{2 r}=\left(4 k^{2}+4 k+1\right)^{r}=\sum_{i=0}^{r}\left(\begin{array}{l}
r \\
i
\end{array}\right) 4^{i} k^{i}(k+1)^{i}
$$

we deduce the congruence (1.4) from (1.3) immediately. 


\section{Proof of Theorem 1.2}

Similarly to the proof of Lemma 2.2, we can check the following result (see [14]).

Lemma 3.1 For all $k, n \in \mathbb{N}$, there hold

$$
\begin{aligned}
\sum_{m=0}^{n-1}(2 m+1)\left(\begin{array}{c}
m+k \\
2 k
\end{array}\right) & =\frac{(n-k) n}{k+1}\left(\begin{array}{c}
n+k \\
2 k
\end{array}\right) \\
\sum_{m=0}^{n-1}(-1)^{m}(2 m+1)\left(\begin{array}{c}
m+k \\
2 k
\end{array}\right) & =(-1)^{n-1}(n-k)\left(\begin{array}{c}
n+k \\
2 k
\end{array}\right) .
\end{aligned}
$$

Proof of Theorem 1.2. Substituting (1.2) for $D_{m}$ and applying Lemmas 2.1 and 3.1, we obtain

$$
\begin{aligned}
& \sum_{m=0}^{n-1}(2 m+1) m^{r}(m+1)^{r} D_{m} \\
& =\sum_{j=0}^{r} \sum_{k=0}^{n-1} a_{j}(k, r)\left(\begin{array}{c}
2 k \\
k
\end{array}\right)(2 k+1)_{2 j} \frac{(n-k-j) n}{k+j+1}\left(\begin{array}{c}
n+k+j \\
2 k+2 j
\end{array}\right) \\
& =n \sum_{j=0}^{r} \sum_{k=0}^{n-1} a_{j}(k, r) \frac{(n-k-j)}{k+j+1}\left(\begin{array}{c}
2 k+2 j \\
k+j
\end{array}\right)(k+1)_{j}^{2}\left(\begin{array}{c}
n+k+j \\
2 k+2 j
\end{array}\right),
\end{aligned}
$$

where $a_{0}(k, r), \ldots, a_{r}(k, r)$ are integers determined by $(2.1)$. Since $\frac{1}{k+j+1}\left(\begin{array}{c}2 k+2 j \\ k+j\end{array}\right)$ is an integer (a Catalan number), we derive (1.5) for $\varepsilon=1$. Similarly, we have

$$
\begin{aligned}
& \sum_{m=0}^{n-1}(-1)^{m}(2 m+1) m^{r}(m+1)^{r} D_{m} \\
& =\sum_{j=0}^{r} \sum_{k=0}^{n-1} a_{j}(k, r)\left(\begin{array}{c}
2 k \\
k
\end{array}\right)(2 k+1)_{2 j}(-1)^{n-1}(n-k-j)\left(\begin{array}{c}
n+k+j \\
2 k+2 j
\end{array}\right) \\
& =\sum_{j=0}^{r} \sum_{k=0}^{n-1} a_{j}(k, r)(-1)^{n-1}(n-k-j)\left(\begin{array}{c}
2 k+2 j \\
k+j
\end{array}\right)(k+1)_{j}^{2}\left(\begin{array}{c}
n+k+j \\
2 k+2 j
\end{array}\right) .
\end{aligned}
$$

Writing $(n-k-j)\left(\begin{array}{c}2 k+2 j \\ k+j\end{array}\right)\left(\begin{array}{c}n+k+j \\ 2 k+2 j\end{array}\right)=n\left(\begin{array}{c}n+k+j \\ n\end{array}\right)\left(\begin{array}{c}n-1 \\ k+j\end{array}\right)$, we obtain (1.5) for $\varepsilon=-1$. Thus, the congruence (1.5) holds for $\varepsilon= \pm 1$. By the relation (2.4), we deduce the congruence (1.6) from (1.5) immediately.

The congruence (1.6) has the following refinements. 
Theorem 3.2 Let $r \in \mathbb{N}$ and $n \in \mathbb{Z}^{+}$. Then

$$
\begin{aligned}
\sum_{k=0}^{n-1}(2 k+1)^{2 r+1} D_{k} & \equiv n \quad(\bmod 2 n), \\
\sum_{k=0}^{n-1}(-1)^{k}(2 k+1)^{2 r+1} D_{k} & \equiv\left\{\begin{array}{ll}
n, & \text { if } n \text { is odd } \\
0, & \text { otherwise }
\end{array} \quad(\bmod 2 n) .\right.
\end{aligned}
$$

Proof. By the relation (2.4) and the congruence (1.5), it is enough to prove the case $r=0$. Substituting (1.2) for $D_{k}$ and exchanging the order of summations, we obtain, by applying Lemma 3.1, that

$$
\begin{gathered}
\sum_{k=0}^{n-1}(2 k+1) D_{k}=n \sum_{k=0}^{n-1}\left(\begin{array}{c}
n+k \\
n
\end{array}\right)\left(\begin{array}{c}
n \\
k+1
\end{array}\right), \\
\sum_{k=0}^{n-1}(-1)^{k}(2 k+1) D_{k}=(-1)^{n} n \sum_{k=0}^{n-1}\left(\begin{array}{c}
n+k \\
n
\end{array}\right)\left(\begin{array}{c}
n-1 \\
k
\end{array}\right),
\end{gathered}
$$

as already mentioned by Sun [14]. Noticing that

$$
\begin{aligned}
& \sum_{k=0}^{n-1}\left(\begin{array}{c}
n+k \\
n
\end{array}\right)\left(\begin{array}{c}
n \\
k+1
\end{array}\right) \equiv \sum_{k=0}^{n-1}(-1)^{k}\left(\begin{array}{c}
n+k \\
n
\end{array}\right)\left(\begin{array}{c}
n \\
k+1
\end{array}\right)=(-1)^{n-1} \quad(\bmod 2) \\
& \sum_{k=0}^{n-1}\left(\begin{array}{c}
n+k \\
n
\end{array}\right)\left(\begin{array}{c}
n-1 \\
k
\end{array}\right) \equiv \sum_{k=0}^{n-1}(-1)^{k}\left(\begin{array}{c}
n+k \\
n
\end{array}\right)\left(\begin{array}{c}
n-1 \\
k
\end{array}\right)=(-1)^{n-1} n \quad(\bmod 2),
\end{aligned}
$$

we have

$$
\begin{aligned}
\sum_{k=0}^{n-1}(2 k+1) D_{k} & \equiv n \quad(\bmod 2 n) \\
\sum_{k=0}^{n-1}(-1)^{k}(2 k+1) D_{k} & \equiv\left\{\begin{array}{ll}
n, & \text { if } n \text { is odd } \\
0, & \text { otherwise }
\end{array} \quad(\bmod 2 n)\right.
\end{aligned}
$$

This completes the proof.

Conjecture 3.3 Let $n$ be a power of 2 . Then

$$
\sum_{k=0}^{n-1}(-1)^{k}(2 k+1)^{3} D_{k} \equiv 2 n^{2} \quad\left(\bmod n^{3}\right) .
$$




\section{Proof of Theorem 1.3}

We first prove two lemmas. The first one can be proved easily by induction on $n$, or be verified automatically in Maple.

Lemma 4.1 For $0 \leqslant k \leqslant n-1$ we have

$$
\sum_{m=0}^{n-1}(2 m+1)^{3}\left(\begin{array}{c}
m+k \\
2 k
\end{array}\right)^{2}=\frac{(n-k)^{2}\left(2 n^{2}-k-1\right)}{k+1}\left(\begin{array}{c}
n+k \\
2 k
\end{array}\right)^{2} .
$$

Lemma 4.2 For any prime $p>3$, we have

$$
\begin{array}{r}
\sum_{k=0}^{p-1}\left(\begin{array}{c}
p+k \\
k
\end{array}\right)^{2}\left(\begin{array}{c}
p-1 \\
k
\end{array}\right)^{2} \equiv p \quad\left(\bmod 2 p^{4}\right), \\
\sum_{k=0}^{p-1}\left(\begin{array}{c}
p+k \\
k+1
\end{array}\right)\left(\begin{array}{c}
p+k \\
k
\end{array}\right)\left(\begin{array}{c}
p-1 \\
k
\end{array}\right)^{2} \equiv 1 \quad\left(\bmod 2 p^{3}\right) .
\end{array}
$$

Proof. For $0 \leqslant k \leqslant p-1$, we have

$$
\begin{aligned}
\left(\begin{array}{c}
p+k \\
k
\end{array}\right)^{2}\left(\begin{array}{c}
p-1 \\
k
\end{array}\right)^{2} & =\prod_{j=1}^{k} \frac{(p+j)^{2}(p-j)^{2}}{j^{4}}=\prod_{j=1}^{k}\left(1-\frac{p^{2}}{j^{2}}\right)^{2} \\
& \equiv 1-2 p^{2} \sum_{j=1}^{k} \frac{1}{j^{2}} \quad\left(\bmod p^{4}\right) .
\end{aligned}
$$

Therefore,

$$
\begin{aligned}
\sum_{k=0}^{p-1}\left(\begin{array}{c}
p+k \\
k
\end{array}\right)^{2}\left(\begin{array}{c}
p-1 \\
k
\end{array}\right)^{2} & \equiv \sum_{k=0}^{p-1}\left(1-2 p^{2} \sum_{j=1}^{k} \frac{1}{j^{2}}\right) \\
& =p-2 p^{2} \sum_{j=1}^{p-1} \frac{p-j}{j^{2}} \quad\left(\bmod p^{4}\right)
\end{aligned}
$$

By Wolstenholme's theorem (see [11]), i.e., for $p>3$,

$$
\sum_{k=1}^{p-1} \frac{1}{k} \equiv 0 \quad\left(\bmod p^{2}\right), \quad \sum_{k=1}^{p-1} \frac{1}{k^{2}} \equiv 0 \quad(\bmod p)
$$

we obtain

$$
\sum_{k=0}^{p-1}\left(\begin{array}{c}
p+k \\
k
\end{array}\right)^{2}\left(\begin{array}{c}
p-1 \\
k
\end{array}\right)^{2} \equiv p \quad\left(\bmod p^{4}\right)
$$


Combining (4.5) and (3.1) with $n=p$, we obtain the congruence (4.1).

Since $\left(\begin{array}{c}p+k \\ k+1\end{array}\right)=\frac{p}{k+1}\left(\begin{array}{c}p+k \\ k\end{array}\right)$, by (4.3) we have

$$
\left(\begin{array}{c}
p+k \\
k+1
\end{array}\right)\left(\begin{array}{c}
p+k \\
k
\end{array}\right)\left(\begin{array}{c}
p-1 \\
k
\end{array}\right)^{2} \equiv\left\{\begin{array}{ll}
1-2 p^{2} \sum_{j=1}^{k} \frac{1}{j^{2}}, & \text { if } k=p-1 \\
\frac{p}{k+1}\left(1-2 p^{2} \sum_{j=1}^{k} \frac{1}{j^{2}}\right) & \text { if } 0 \leqslant k \leqslant p-2
\end{array} \quad\left(\bmod p^{4}\right)\right.
$$

and hence by (4.4) we get

$$
\left(\begin{array}{c}
p+k \\
k+1
\end{array}\right)\left(\begin{array}{c}
p+k \\
k
\end{array}\right)\left(\begin{array}{c}
p-1 \\
k
\end{array}\right)^{2} \equiv\left\{\begin{array}{ll}
1, & \text { if } k=p-1 \\
\frac{p}{k+1} & \text { if } 0 \leqslant k \leqslant p-2
\end{array} \quad\left(\bmod p^{3}\right) .\right.
$$

It follows that, for $p>3$,

$$
\sum_{k=0}^{p-1}\left(\begin{array}{c}
p+k \\
k+1
\end{array}\right)\left(\begin{array}{c}
p+k \\
k
\end{array}\right)\left(\begin{array}{c}
p-1 \\
k
\end{array}\right)^{2} \equiv 1+\sum_{k=0}^{p-2} \frac{p}{k+1} \equiv 1 \quad\left(\bmod p^{3}\right)
$$

by (4.4). On the other hand, we have

$$
\begin{aligned}
\sum_{k=0}^{p-1}\left(\begin{array}{c}
p+k \\
k+1
\end{array}\right)\left(\begin{array}{c}
p+k \\
k
\end{array}\right)\left(\begin{array}{c}
p-1 \\
k
\end{array}\right)^{2} & \equiv \sum_{k=0}^{p-1}\left(\begin{array}{c}
p+k \\
k+1
\end{array}\right)\left(\begin{array}{c}
p+k \\
k
\end{array}\right)\left(\begin{array}{c}
p-1 \\
k
\end{array}\right) \\
& =\sum_{k=0}^{p-1}\left(\begin{array}{c}
p+k \\
k
\end{array}\right)^{2}\left(\begin{array}{c}
p \\
k+1
\end{array}\right) \\
& \equiv \sum_{k=0}^{p-1}(-1)^{k}\left(\begin{array}{c}
p+k \\
k
\end{array}\right)\left(\begin{array}{c}
p \\
k+1
\end{array}\right)=(-1)^{p-1} \quad(\bmod 2) .
\end{aligned}
$$

Combining (4.6) and (4.7), we obtain the congruence (4.2).

Proof of Theorem 1.3. Substituting (1.1) for $A_{m}$ and exchanging the order of summations, by Lemma 4.1, we obtain

$$
\begin{aligned}
\sum_{m=0}^{n-1}(2 m+1)^{3} A_{m} & =\sum_{k=0}^{n-1}\left(\begin{array}{c}
2 k \\
k
\end{array}\right)^{2} \frac{(n-k)^{2}\left(2 n^{2}-k-1\right)}{k+1}\left(\begin{array}{c}
n+k \\
2 k
\end{array}\right)^{2} \\
& =\sum_{k=0}^{n-1}\left(\begin{array}{c}
n+k \\
k
\end{array}\right)^{2} \frac{(n-k)^{2}\left(2 n^{2}-k-1\right)}{k+1}\left(\begin{array}{c}
n \\
k
\end{array}\right)^{2} \\
& =2 n^{3} \sum_{k=0}^{n-1}\left(\begin{array}{c}
n+k \\
k+1
\end{array}\right)\left(\begin{array}{c}
n+k \\
k
\end{array}\right)\left(\begin{array}{c}
n-1 \\
k
\end{array}\right)^{2}-n^{2} \sum_{k=0}^{n-1}\left(\begin{array}{c}
n+k \\
k
\end{array}\right)^{2}\left(\begin{array}{c}
n-1 \\
k
\end{array}\right)^{2} .
\end{aligned}
$$

Applying (1.9) to (4.8), we immediately get the congruence (1.7). Now, assume that $n=p>3$ is a prime in (4.8). Then the congruence (1.8) follows from (4.1) and (4.2). 


\section{Proof of Theorem 1.4}

We shall prove several $q$-versions of (1.10). We first recall some $q$-notations and two fundamental results. The $q$-binomial coefficients and $q$-integers are polynomials in $q$ with integer coefficients and are defined by

$$
\left[\begin{array}{l}
n \\
k
\end{array}\right]_{q}=\prod_{j=1}^{k} \frac{1-q^{n-j+1}}{1-q^{j}}, \quad[n]_{q}=\frac{1-q^{n}}{1-q} .
$$

The following two results are well known (see [1, (3.3.10)] and [8,10,12]).

Lemma 5.1 (The q-Chu-Vandermonde formula) For nonnegative integers $m, n$ and $h$ there holds

$$
\sum_{k=0}^{h}\left[\begin{array}{l}
n \\
k
\end{array}\right]_{q}\left[\begin{array}{c}
m \\
h-k
\end{array}\right]_{q} q^{(n-k)(h-k)}=\left[\begin{array}{c}
m+n \\
h
\end{array}\right]_{q} .
$$

Lemma 5.2 (The $q$-Lucas Theorem) Let $a, b, r, s$ and $n$ be nonnegative integers such that $0 \leqslant b, s \leqslant n-1$. Then, for any nth primitive root of unity $\omega$, there holds

$$
\left[\begin{array}{l}
a d+b \\
r d+s
\end{array}\right]_{\omega}=\left(\begin{array}{l}
a \\
r
\end{array}\right)\left[\begin{array}{l}
b \\
s
\end{array}\right]_{\omega}
$$

Given three polynomials $f(x), g(x)$ and $q(x)$ in $\mathbb{Z}[x]$, if $q(x)$ divides $f(x)-g(x)$ in $\mathbb{Z}[x]$ we write $f(x) \equiv g(x)(\bmod q(x))$. We shall prove the following $q$-versions of Theorem 1.4.

Theorem 5.3 For any nonnegative integers $a_{1}, \ldots, a_{m}, b_{1}, \ldots, b_{m}$ and positive integer $n$, let $d:=\operatorname{gcd}\left(a_{1}, \ldots, a_{m}, b_{1}, \ldots, b_{m}, n\right)$. Then

$$
\begin{gathered}
\sum_{k=0}^{n-1} q^{k^{2}}\left[\begin{array}{c}
n-1 \\
k
\end{array}\right]_{q}^{2} \prod_{i=1}^{m}\left[\begin{array}{l}
a_{i}+k \\
b_{i}+k
\end{array}\right]_{q} \equiv 0 \quad\left(\bmod [d]_{q}\right), \\
\sum_{k=0}^{n-1} q^{k^{2}+2 k}\left[\begin{array}{c}
n-1 \\
k
\end{array}\right]_{q}^{2} \prod_{i=1}^{m}\left[\begin{array}{l}
a_{i}+k \\
b_{i}+k
\end{array}\right]_{q} \equiv 0 \quad\left(\bmod [d]_{q}\right), \\
\sum_{k=0}^{n-1} q^{k} \prod_{i=1}^{m}\left[\begin{array}{l}
a_{i}+k \\
b_{i}+k
\end{array}\right]_{q} \equiv 0 \quad\left(\bmod [d]_{q}\right), \\
\sum_{k=0}^{n-1} q^{n-k-1} \prod_{i=1}^{m}\left[\begin{array}{l}
a_{i}+k \\
b_{i}+k
\end{array}\right]_{q} \equiv 0 \quad\left(\bmod [d]_{q}\right),
\end{gathered}
$$

Proof. Let $d_{1} \in \mathbb{Z}^{+}$such that $d_{1} \mid d$ and $d_{1}>1$, and let

$$
a_{i}=a_{i}^{\prime} d_{1}, b_{i}=b_{i}^{\prime} d_{1}, i=1, \ldots, m, \text { and } n=n^{\prime} d_{1} \text {. }
$$


Suppose that $\omega$ is a primitive $d_{1}$-th root of unity. Replacing $k$ by $j d_{1}+k$ with $0 \leqslant j \leqslant n^{\prime}-1$ and $0 \leqslant k \leqslant d_{1}-1$, we obtain, by the $q$-Lucas theorem,

$$
\begin{aligned}
\sum_{k=0}^{n-1} \omega^{k^{2}}\left[\begin{array}{c}
n-1 \\
k
\end{array}\right]_{\omega}^{2} \prod_{i=1}^{m}\left[\begin{array}{c}
a_{i}+k \\
b_{i}+k
\end{array}\right]_{\omega} & =\sum_{j=0}^{n^{\prime}-1} \sum_{k=0}^{d_{1}-1} \omega^{\left(j d_{1}+k\right)^{2}}\left[\begin{array}{c}
n^{\prime} d_{1}-1 \\
j d_{1}+k
\end{array}\right]_{\omega}^{2} \prod_{i=1}^{m}\left[\begin{array}{c}
a_{i}^{\prime} d_{1}+j d_{1}+k \\
b_{i}^{\prime} d_{1}+j d_{1}+k
\end{array}\right]_{\omega} \\
& =\sum_{j=0}^{n^{\prime}-1} \sum_{k=0}^{d_{1}-1} \omega^{k^{2}}\left(\begin{array}{c}
n^{\prime}-1 \\
j
\end{array}\right)^{2}\left[\begin{array}{c}
d_{1}-1 \\
k
\end{array}\right]_{\omega}^{2} \prod_{i=1}^{m}\left(\begin{array}{c}
a_{i}^{\prime}+j \\
b_{i}^{\prime}+j
\end{array}\right) \\
& =\left(\sum_{j=0}^{n^{\prime}-1}\left(\begin{array}{c}
n^{\prime}-1 \\
j
\end{array}\right)^{2} \prod_{i=1}^{m}\left(\begin{array}{c}
a_{i}^{\prime}+j \\
b_{i}^{\prime}+j
\end{array}\right)\right)^{d_{1}-1} \sum_{k=0}^{k^{2}}\left[\begin{array}{c}
d_{1}-1 \\
k
\end{array}\right]_{\omega}^{2} .
\end{aligned}
$$

By applying the $q$-Chu-Vandermonde formula and the $q$-Lucas theorem, we have

$$
\sum_{k=0}^{d_{1}-1} \omega^{k^{2}}\left[\begin{array}{c}
d_{1}-1 \\
k
\end{array}\right]_{\omega}^{2}=\left[\begin{array}{c}
2 d_{1}-2 \\
d_{1}-1
\end{array}\right]_{\omega}=\left[\begin{array}{c}
d_{1}-2 \\
d_{1}-1
\end{array}\right]_{\omega}=0
$$

This proves that

$$
\sum_{k=0}^{n-1} q^{k^{2}}\left[\begin{array}{c}
n-1 \\
k
\end{array}\right]_{q}^{2} \prod_{i=1}^{m}\left[\begin{array}{c}
a_{i}+k \\
b_{i}+k
\end{array}\right]_{q} \equiv 0 \quad\left(\bmod \Phi_{d_{1}}(q)\right) \quad \text { for any } d_{1} \mid d \text { and } d_{1}>1
$$

where $\Phi_{n}(q)$ is the $n$th cyclotomic polynomial in $q$. Since

$$
\frac{1-q^{d}}{1-q}=\prod_{\substack{d_{1} \mid d \\ d_{1}>1}} \Phi_{d_{1}}(q)
$$

and the cyclotomic polynomials are pairwise relatively prime, we complete the proof of (5.2). Similarly, observing that

$$
\begin{gathered}
\sum_{k=0}^{d_{1}-1} \omega^{k^{2}+2 k}\left[\begin{array}{c}
d_{1}-1 \\
k
\end{array}\right]_{\omega}^{2}=\omega^{-1} \sum_{k=0}^{d_{1}-1} \omega^{\left(d_{1}-k-1\right)^{2}}\left[\begin{array}{c}
d_{1}-1 \\
k
\end{array}\right]_{\omega}^{2}=\omega^{-1}\left[\begin{array}{c}
2 d_{1}-2 \\
d_{1}-1
\end{array}\right]_{\omega}=0 \\
\sum_{k=0}^{d_{1}-1} \omega^{k}=\sum_{k=0}^{d_{1}-1} \omega^{-k-1}=0
\end{gathered}
$$

we can prove $(5.3)-(5.5)$.

To derive more consequences of Theorem 5.3, we need the following lemma.

Lemma 5.4 Let $P(x) \in \mathbb{Z}[x]$ and $d \in \mathbb{Z}^{+}$. If $P(n) \equiv 0(\bmod d)$ for all $n \in \mathbb{N}$, then $P(m) \equiv 0(\bmod d)$ for all $m \in \mathbb{Z}$.

Proof. Just notice that $P(m+k d) \equiv P(m)(\bmod d)$ for all $m, k \in \mathbb{Z}$.

We now give the following generalization of Theorem 1.4. 
Theorem 5.5 Let $a_{1}, \ldots, a_{m} \in \mathbb{Z}, b_{1}, \ldots, b_{m} \in \mathbb{N}$ and $n \in \mathbb{Z}^{+}$. Then

$$
\sum_{k=0}^{n-1}(-1)^{m k} \prod_{i=1}^{m}\left(\begin{array}{l}
a_{i}-1 \\
b_{i}+k
\end{array}\right) \equiv 0 \quad\left(\bmod \operatorname{gcd}\left(a_{1}, \ldots, a_{m}, b_{1}, \ldots, b_{m}, n\right)\right) .
$$

Proof. Suppose that $d$ is a factor of $n$. Letting $q=1$ in (5.4), we obtain

$$
\sum_{k=0}^{n-1} \prod_{i=1}^{m}\left(\begin{array}{l}
x_{i} d+k \\
y_{i} d+k
\end{array}\right) \equiv 0 \quad(\bmod d)
$$

for all $x_{1}, \ldots, x_{m}, y_{1}, \ldots, y_{m} \in \mathbb{N}$. Since $P\left(x_{1}\right):=\sum_{k=0}^{n-1} \prod_{i=1}^{m}\left(\begin{array}{l}x_{i} d+k \\ y_{i} d+k\end{array}\right)$ is a polynomial in $x_{1}$ with rational coefficients, there is a positive integer $\alpha$ such that $\alpha P\left(x_{1}\right) \in \mathbb{Z}\left[x_{1}\right]$. Then (5.6) is equivalent to

$$
\alpha P\left(x_{1}\right) \equiv 0 \quad(\bmod \alpha d) .
$$

By Lemma 5.4, we see that (5.7) is true for all $x_{1} \in \mathbb{Z}$, and so is (5.6). By symmetry, we conclude that (5.6) holds for all $x_{1}, \ldots, x_{m} \in \mathbb{Z}$. Namely, for all $a_{1}, \ldots, a_{m} \in \mathbb{Z}$, $b_{1}, \ldots, b_{m} \in \mathbb{N}$, there holds

$$
\sum_{k=0}^{n-1} \prod_{i=1}^{m}\left(\begin{array}{c}
b_{i}-a_{i}+k \\
b_{i}+k
\end{array}\right) \equiv 0 \quad\left(\bmod \operatorname{gcd}\left(a_{1}, \ldots, a_{m}, b_{1}, \ldots, b_{m}, n\right)\right) .
$$

Noticing that $\left(\begin{array}{c}b_{i}-a_{i}+k \\ b_{i}+k\end{array}\right)=(-1)^{b_{i}+k}\left(\begin{array}{c}a_{i}-1 \\ b_{i}+k\end{array}\right)$, we complete the proof.

Letting $b_{i}=0$ and $a_{i}= \pm n$ in Theorem 5.5, we obtain

Corollary 5.6 Let $r, s, n \in \mathbb{N}$. Then

$$
\begin{array}{r}
\sum_{k=0}^{n-1}\left(\begin{array}{c}
n+k \\
k
\end{array}\right)^{r}\left(\begin{array}{c}
n-1 \\
k
\end{array}\right)^{2 s} \equiv 0 \quad(\bmod n), \\
\sum_{k=0}^{n-1}(-1)^{k}\left(\begin{array}{c}
n+k \\
k
\end{array}\right)^{r}\left(\begin{array}{c}
n-1 \\
k
\end{array}\right)^{2 s+1} \equiv 0 \quad(\bmod n),
\end{array}
$$

In particular, we have

$$
\sum_{k=0}^{n}\left(\begin{array}{l}
n \\
k
\end{array}\right)^{2 s} \equiv 0 \quad(\bmod (n+1))
$$

It is worth mentioning that Calkin [4, Proposition 3] has proved that

$$
\sum_{k=0}^{n}\left(\begin{array}{l}
n \\
k
\end{array}\right)^{2 s} \equiv 0 \quad(\bmod p)
$$

if $p$ is a prime such that $\frac{n}{m}<p<\frac{n+1}{m}+\frac{n+1-m}{m(2 m s-1)}$ for some $m \in \mathbb{Z}^{+}$. It is clear that (5.8) and (5.9) are different and can not be deduced from each other.

Letting $b_{i}=0$ and $a_{i}=-n$ or $a_{i}=-2 n$ in Theorem 5.5, we obtain 
Corollary 5.7 Let $r, s, n \in \mathbb{N}$. Then

$$
\sum_{k=0}^{n-1}(-1)^{(r+s) k}\left(\begin{array}{c}
n-1 \\
k
\end{array}\right)^{r}\left(\begin{array}{c}
2 n-1 \\
k
\end{array}\right)^{s} \equiv 0 \quad(\bmod n) .
$$

Note that Chamberland and Dilcher [6] have studied divisibility properties of the following similar sums:

$$
\sum_{k=0}^{n} \varepsilon^{k}\left(\begin{array}{l}
n \\
k
\end{array}\right)^{r}\left(\begin{array}{c}
2 n \\
k
\end{array}\right)^{s}
$$

where $\varepsilon= \pm 1$.

Conjecture 5.8 Let $a_{1}, \ldots, a_{m} \in \mathbb{Z}, b_{1}, \ldots, b_{m}, r \in \mathbb{N}$ and $n \in \mathbb{Z}^{+}$. Then

$$
\sum_{k=0}^{n-1}(-1)^{m k} \varepsilon^{k}(2 k+1) k^{r}(k+1)^{r} \prod_{i=1}^{m}\left(\begin{array}{c}
a_{i}-1 \\
b_{i}+k
\end{array}\right) \equiv 0 \quad\left(\bmod \operatorname{gcd}\left(a_{1}, \ldots, a_{m}, b_{1}, \ldots, b_{m}, n\right)\right),
$$

where $\varepsilon= \pm 1$.

Conjecture 5.9 Let $n, r \in \mathbb{Z}^{+}$. Then

$$
\begin{aligned}
\sum_{k=0}^{n-1}\left(\begin{array}{c}
n-1 \\
k
\end{array}\right)^{2 r} & \equiv\left\{\begin{array}{ll}
n, & \text { if } n=2^{a} \\
0, & \text { otherwise }
\end{array} \quad(\bmod 2 n),\right. \\
\sum_{k=0}^{n-1}\left(\begin{array}{c}
2 n-1 \\
k
\end{array}\right)^{2 r} & \equiv\left\{\begin{array}{ll}
n, & \text { if } n=2^{a} \\
0, & \text { otherwise }
\end{array} \quad(\bmod 2 n) .\right.
\end{aligned}
$$

Conjecture 5.10 Let $n, r \in \mathbb{N}$ and $s, t \in \mathbb{Z}^{+}$. Then

$$
\sum_{k=0}^{n-1}(-1)^{k t}\left(\begin{array}{c}
n+k \\
k
\end{array}\right)^{s}\left(\begin{array}{c}
n-1 \\
k
\end{array}\right)^{t} \equiv\left\{\begin{array}{ll}
0, & \text { if } 2 \mid n \text { and } 2 \nmid(s+t) \\
n, & \text { otherwise }
\end{array} \quad(\bmod 2 n) .\right.
$$

Lemma 5.11 Let $n \in \mathbb{N}$. Then

$$
\sum_{k=0}^{n}\left[\begin{array}{l}
n \\
k
\end{array}\right]_{q}^{2} q^{k^{2}-k}=2\left[\begin{array}{c}
2 n-1 \\
n
\end{array}\right]_{q}
$$

Proof. By the $q$-Chu-Vandermonde formula (5.1), we have

$$
\begin{aligned}
\sum_{k=0}^{n}\left[\begin{array}{l}
n \\
k
\end{array}\right]_{q}\left[\begin{array}{c}
n-1 \\
k
\end{array}\right]_{q} q^{k^{2}} & =\left[\begin{array}{c}
2 n-1 \\
n
\end{array}\right]_{q}, \\
\sum_{k=0}^{n}\left[\begin{array}{l}
n \\
k
\end{array}\right]_{q}\left[\begin{array}{l}
n-1 \\
k-1
\end{array}\right]_{q} q^{k(k-1)} & =\left[\begin{array}{c}
2 n-1 \\
n
\end{array}\right]_{q} .
\end{aligned}
$$

The result then follows from the relation $\left[\begin{array}{c}n \\ k\end{array}\right]_{q}=\left[\begin{array}{c}n-1 \\ k\end{array}\right]_{q} q^{k}+\left[\begin{array}{c}n-1 \\ k-1\end{array}\right]_{q}$. 
Theorem 5.12 Let $n \in \mathbb{Z}^{+}$. Then

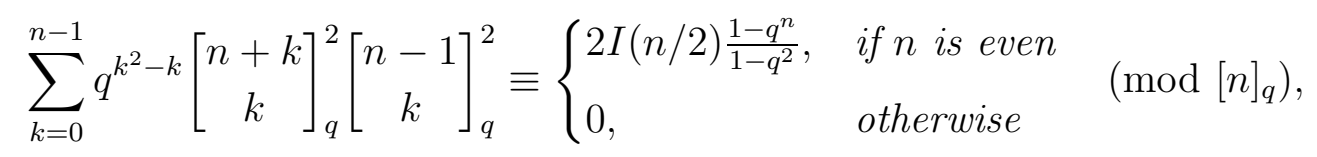

where $I(n)=\frac{1}{n} \sum_{k=0}^{n-1}\left(\begin{array}{c}n+k \\ k\end{array}\right)^{2}\left(\begin{array}{c}n-1 \\ k\end{array}\right)^{2}$.

Proof. Let $d_{1} \mid n\left(d_{1}>1\right)$ and $n^{\prime}=n / d_{1}$. Suppose that $\omega$ is a primitive $d_{1}$-th root of unity. Similarly to the proof of Theorem 5.3, we have

$$
\begin{aligned}
\sum_{k=0}^{n-1} \omega^{k^{2}-k}\left[\begin{array}{c}
n-1 \\
k
\end{array}\right]_{\omega}^{2}\left[\begin{array}{c}
n+k \\
k
\end{array}\right]_{\omega}^{2} & =\sum_{j=0}^{n^{\prime}-1}\left(\begin{array}{c}
n^{\prime}-1 \\
j
\end{array}\right)^{2}\left(\begin{array}{c}
n^{\prime}+j \\
j
\end{array}\right)^{2} \sum_{k=0}^{d_{1}-1} \omega^{k^{2}-k}\left[\begin{array}{c}
d_{1}-1 \\
k
\end{array}\right]_{\omega}^{2} \\
& =2\left[\begin{array}{c}
2 d_{1}-3 \\
d_{1}-1
\end{array}\right]_{\omega} \sum_{j=0}^{n^{\prime}-1}\left(\begin{array}{c}
n^{\prime}-1 \\
j
\end{array}\right)^{2}\left(\begin{array}{c}
n^{\prime}+j \\
j
\end{array}\right)^{2} \text { by (5.10). }
\end{aligned}
$$

If $n$ is odd, then $d_{1} \geqslant 3$, and by the $q$-Lucas theorem,

$$
\left[\begin{array}{c}
2 d_{1}-3 \\
d_{1}-1
\end{array}\right]_{\omega}=\left[\begin{array}{l}
d_{1}-3 \\
d_{1}-1
\end{array}\right]_{\omega}=0
$$

which means that

$$
\sum_{k=0}^{n-1} q^{k^{2}-k}\left[\begin{array}{c}
n+k \\
k
\end{array}\right]_{q}^{2}\left[\begin{array}{c}
n-1 \\
k
\end{array}\right]_{q}^{2} \equiv 0 \quad\left(\bmod [n]_{q}\right)
$$

If $n$ is even, then it is easy to check that (for $d_{1}=2$ or $d_{1} \geqslant 3$ )

$$
\sum_{k=0}^{n-1} \omega^{k^{2}-k}\left[\begin{array}{c}
n-1 \\
k
\end{array}\right]_{\omega}^{2}\left[\begin{array}{c}
n+k \\
k
\end{array}\right]_{\omega}^{2}-2 I(n / 2) \sum_{k=0}^{(n-2) / 2} \omega^{2 k}=0
$$

which implies the first congruence in (5.11). This completes the proof.

Conjecture 5.13 Let $n$ be any power of a prime $p$. Then

$$
\sum_{k=0}^{n-1} q^{(n-k)^{2}}\left[\begin{array}{c}
n+k \\
k
\end{array}\right]_{q}^{2}\left[\begin{array}{c}
n-1 \\
k
\end{array}\right]_{q}^{2} \equiv q^{(n-1)^{2}}[n]_{q} \quad\left(\bmod [p]_{q^{n / p}}^{2}\right) .
$$

It is easy to see that Conjecture 5.13 is true for $q=1$ by the congruences (1.9) and $(4.1)$.

Acknowledgments. This work was partially supported by the Fundamental Research Funds for the Central Universities, Shanghai Rising-Star Program (\#09QA1401700) and the National Science Foundation of China (\#10801054). 


\section{References}

[1] G.E. Andrews, The Theory of Partitions, Cambridge University Press, Cambridge, 1998.

[2] R. Apéry, Irrationalité de $\zeta(2)$ et $\zeta(3)$, Astérisque 61 (1979), 11-13.

[3] F. Beukers, Another congruence for the Apéry numbers, J. Number Theory 25 (1987), 201-210.

[4] N.J. Calkin, Factors of sums of powers of binomial coefficients, Acta Arith. 86 (1998), $17-26$.

[5] J.S. Caughman, C.R. Haithcock and J.J.P. Veerman, A note on lattice chains and Delannoy numbers, Discrete Math. 308 (2008), 2623-2628.

[6] M. Chamberland and K. Dilcher, Divisibility properties of a class of binomial sums, J. Number Theory 120 (2006), 349-371.

[7] S. Chowla, J. Cowles and M. Cowles, Congruence properties of Apéry numbers, J. Number Theory 12 (1980), 188-190.

[8] J. Désarménien, Un analogue des congruences de Kummer pour les $q$-nombres d'Euler, European J. Combin. 3 (1982), 19-28.

[9] I. Gessel, Some congruences for Apéry Numbers, J. Number Theory 14 (1982), 362-368.

[10] V.J.W. Guo and J. Zeng, Some arithmetic properties of the $q$-Euler numbers and $q$-Salié numbers, European J. Combin. 27 (2006), 884-895.

[11] G.H. Hardy and E.M. Wright, An Introduction to the Theory of Numbers, 5th Editon, the Clarendon Press, Oxford University Press, New York, 1979, p. 88.

[12] G. Olive, Generalized powers, Amer. Math. Monthly 72 (1965), 619-627.

[13] R.A. Sulanke, Objects counted by the central Delannoy numbers, J. Integer Seq. 6 (2003), Article 03.1.5.

[14] Z.-W. Sun, On sums of Apéry polynomials and related congruences, preprint, arXiv:1101.1946v3.

[15] Z.-W. Sun, Open conjectures on congruences, preprint, arXiv:0911.5665v39. 